\title{
Application of nonsense-mediated primer exclusion (NOPE) for preparation of unique molecular barcoded libraries
}

\author{
Dmitriy A. Shagin ${ }^{1,2+}$, Maria A. Turchaninova ${ }^{1,2,4 \dagger}$, Irina A. Shagina ${ }^{1,2}$, Mikhail Shugay ${ }^{1,2,4}$, Andrew R. Zaretsky ${ }^{1,3}$,
} Olga I. Zueva2, Dmitriy A. Bolotin ${ }^{1,2}$, Sergey Lukyanov ${ }^{1,2}$ and Dmitriy M. Chudakov $1,5,2,4^{*}$

\begin{abstract}
Background: Recently we proposed efficient method to exclude undesirable primers at any stage of amplification reaction, here termed NOPE (NOnsense-mediated Primer Exclusion). According to this method, added oligonucleotide overlapping with the 3'-end of unwanted amplification primer (NOPE oligo) simultaneously provides a template for its elongation. This elongation disrupts specificity of unwanted primer, preventing its further participation in PCR. The suggested approach allows to rationally manage the course of PCR reactions in order to facilitate analysis of complex DNA mixtures as well as to perform multistage PCR bypassing intermediate purification steps.

Results: Here we apply NOPE method to DNA library preparation for the high-throughput sequencing (HTS) with the PCR-based introduction of unique molecular identifiers (UMI). We show that NOPE oligo efficiently neutralizes UMIcontaining oligonucleotides after introduction of UMI into sample DNA molecules, thus allowing to proceed with further amplification steps without purification and associated loss of starting material. At the same time, NOPE oligo does not affect the efficiency of target PCR amplification.

Conclusion: We describe a simple, robust and cheap modification of UMI-labeled HTS libraries preparation procedure, that allows to bypass purification step and thus to preserve starting material which may be limited, e.g. circulating tumor DNA, circulating fetal DNA, or small amounts of isolated cells of interest. Furthermore, demonstrated simplicity and robustness of NOPE method should make it popular in various PCR protocols.
\end{abstract}

Keywords: High-throughput sequencing, Unique molecular identifiers, Targeted resequencing, PCR

\section{Background}

Unique molecular identifiers (UMI) have revolutionized high-throughput sequencing (HTS) strategies. In UMI approach [1-3], each DNA or RNA molecule of a sample gets a unique label at the very first step of library preparation. Once encoded by UMIs, the original sample can be amplified, while the ability to track and distinguish the primary templates through library preparation and sequencing is retained.

UMI provides unprecedented enhancement of the downstream HTS data analysis, by allowing to quantify starting molecules, to eliminate quantitative biases caused

\footnotetext{
* Correspondence: chudakovdm@mail.ru

${ }^{\dagger}$ Equal contributors

${ }^{1}$ Pirogov Russian National Research Medical University, Moscow, Russia

${ }^{5}$ Skolkovo Institute of Science and Technology, Moscow, Russia

Full list of author information is available at the end of the article
}

by PCR and sequencing, to normalize data for comparative analysis, and to eliminate accumulated PCR and sequencing errors. UMI approach is particularly important in reliable detection of rare gene variants in complex libraries. It can be extremely useful in various targeted resequencing applications, such as cancer [4] and prenatal [5] diagnostics, analysis of heterogeneity and variability of tumors [6], bacterial [7] and viral [8] infections, microbiomes [9], and evolutionary studies [10].

Although UMI is a very promising tool for dealing with HTS libraries for targeted resequencing, there are some technical issues that may complicate their use in practical applications. In particular, it is critical that each UMI must be incorporated into DNA or cDNA template molecule only at the very first stage of reaction, in order to uniquely label the true starting molecule. It means 
that UMI-containing primers or ligated adaptors should only be active in the first cycle of PCR, or during cDNA synthesis, or during adaptors ligation. Participation of UMI-containing oligonucleotides in subsequent PCR cycles in the course of library preparation would create artificial "diversity" of starting molecules, undermining the following analysis algorithms. Thus application of UMI in HTS library preparation procedure necessarily requires complete removal of UMI-containing oligonucleotides from the reaction before proceeding to the PCR amplification.

Several approaches employing selective removal or suppression of unwanted priming oligonucleotides in the course of PCR amplification have been developed. Column-based purification, SPRI (solid phase reversible immobilization)-based purification, or enzymatic digestion (uracil-DNA-glycosylase, exonuclease) which also requires purification step, can be used, but all these methods are associated with potential loss of material.

In those situations when starting material is limited, it is preferable to proceed to the PCR without purification. A wide range of blocked oligonucleotides can be used to inhibit undesired PCR amplification without purification stage. Those can be oligonucleotides that are modified so that they do not serve as an initiation point for polymerization of a complementary strand by Taq polymerase or similar enzymes. A number of modifications were suggested to inactivate oligonucleotides, including 3 '-phosphate group [11], and chemically reversed 3 '-terminal nucleotide $\left(3^{\prime}\right.$ to $\left.5^{\prime}\right) /$ inverted end [12] peptide nucleic acids (PNAs), locked nucleic acids (LNAs) etc. Such modified oligonucleotides may compete with the undesirable amplification primers for annealing site or prevent elongation by binding onto the target fragment between the two amplification primers [13-19]. However, these techniques can be implemented only for suppression of external primers before proceeding to internal primers during nested PCR. In the case of step-out PCR, as it is required when dealing with UMI, such blocking oligonucleotides would interfere with subsequent amplification process.

Recently we reported the method for selective, efficient, purification-free neutralization of undesirable primers in PCR reactions [20], here called NOPE (NOnsense-mediated Primer Exclusion, Fig. 1). NOPE approach was successfully applied by another research group for the same application as in our primary work - suppression of megaprimers in alpha-beta TCR pairs matching by overlapextension [21]. Furthermore, NOPE can be also used for a wide variety of applications where it is needed to block undesirable primers or mega-primers. In particular, it can be employed in multistage experiments for blocking the priming activity of DNA molecules remaining from previous reaction stage, e.g. for nested PCR, step-out PCR, PCR after adaptors ligation, etc.
Here we applied NOPE for neutralization of UMIcontaining primer remaining after its PCR-based incorporation into template DNA molecules. We found that addition of NOPE oligo allows to achieve nearly complete elimination of remaining UMI-containing primers without cleanup step. All alternative techniques require more handling or have a higher cost compared to NOPE. In those cases where purification of reaction mixture is impossible/highly undesirable due to the rarity of samples, NOPE becomes an indispensable tool.

\section{Results}

To demonstrate the reliability of NOPE in targeted resequencing, we designed an exemplary experiment, where NOPE oligo was used for elimination of UMI-containing primer after introduction of UMI into the template DNA via linear PCR, and before further amplification (Fig. 2). This experimental system was further employed in our current project on identification of tumor mutations with targeted resequencing of EGFR (epidermal growth factor receptor) gene. Mutations in tyrosine kinase domain of EGFR gene (18-22 exons) are important predictive markers for clinical benefit from EGFR tyrosine kinase inhibitor (TKI) therapies in development for non-small cell lung cancer (NSCLC), one of the most common and deadly cancer [22]. Peripheral blood mononuclear cell (PBMC) DNA of a healthy donor was used in model experiments for demonstration of NOPE oligo efficiency. UMI-barcoding of analyzed DNA was performed using linear PCR followed by amplification of exon 20 human EGFR gene. In this design, efficient neutralization of the UMI-containing primer EGFRex20_NNN by a NOPE oligo should prevent further amplification of the target template with a pair of EGFRex20_NNN and EGFR-ex20_R1 primers, while amplification using a step-out TruSeq-short primer and EGFRex20_R1 primer should remain efficient (Fig. 3). Oligonucleotides used in our study are listed in Table 1.

\section{Design of NOPE oligo}

NOPE oligo should be modified at its 3 '-end, resulting in full inhibition of its enzymatic elongation. Modification must be stable and resistant to degradation or enzymatic removing after synthesis. Earlier we tried several variants of the 3' end modification (Ref. [20] and unpublished data) and finally opted for black hole quencher 1 (BHQ1), which prevents elongation without noticeably influencing the oligos annealing properties.

NOPE oligo may overlap completely with the undesired primer or only partially with its 3 '-end. The length and content of a "nonsense" template (5'-end of a NOPE oligo that serves for the elongation and neutralization of the unwanted primer) may also vary. 


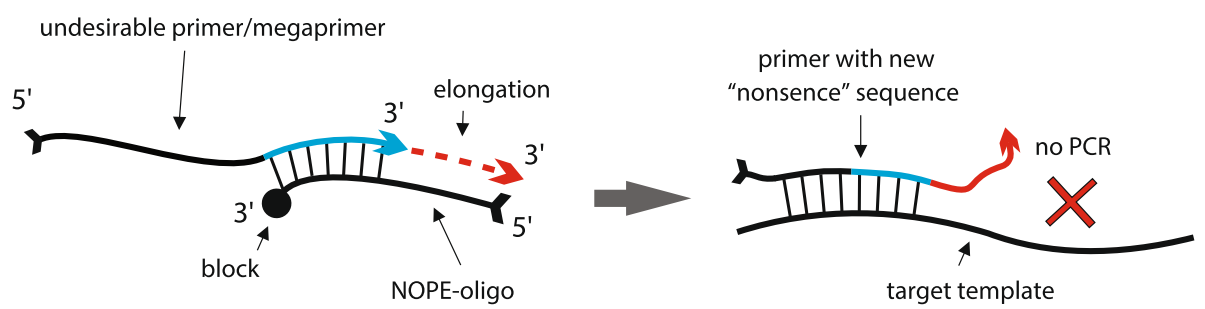

Fig. 1 General principle of NOPE method. NOPE oligo is complementary to the $3^{\prime}$ end of the unwanted primer. Upon binding to the $3^{\prime}$ end of the primer, NOPE oligo provides a template for the primer extension, thereby producing new "nonsense" $3^{\prime}$-end (i.e. the $3^{\prime}$-end that is noncomplementary to the amplified template)

To get better understanding of how efficiency of a NOPE oligo depends on its structure, we tested different possibilities for 3'-overlap and 5'-extension. We designed four different NOPE oligos for neutralization of UMI-containing EGFR-ex20_NNN primer: NOPE-R1, NOPE-R2, NOPE-R3, and NOPE-R0. The latter oligo completely lacked the part for the "nonsense" elongation of the EGFR-ex20_NNN primer. Region of human EGFR gene sequence showing the location of UMI-containing primer and four different NOPE oligos is presented on the Fig. 4.

EGFR gene fragment was amplified as described on Fig. 2. EGFR-ex20_NNN primer neutralization step was performed using one of the NOPE oligos. To detect the minimal NOPE oligos concentration required for efficient elimination of the EGFR-ex20_NNN primer present at concentration $0.1 \mu \mathrm{M}$, we carried out a set of neutralisation PCRs with NOPE oligos concentrations ranging from 0 to $1.5 \mu \mathrm{M}$. After neutralization step, all reactions were divided into two halves (Fig. 3). One half was subsequently amplified (1st PCR) using step-out TruSeq-short primer and reverse primer EGFR-ex20_R1 and showed the overall efficiency of amplification (control). The other half was amplified using only one reverse EGFR-ex20_R1 primer, and reflected the completeness of EGFR-ex20_NNN elimination by a NOPE oligo (test).

Real-time PCR analysis performed with EGFR exon 20 specific TaqMan probe showed that primer exclusion by NOPE-R0 oligo which completely lacks the 5 '-nonsense sequence was inefficient (Figs. 5a,b and 6). The difference in the number of amplification cycles for the PCR with and without TruSeq-short primer was only about 2-3 cycles. This demonstrated that effect of neutralisation based solely on the competition for the EGFRex20_NNN annealing between the template and blocking oligo is minimal.

At the same time, all three blocking oligos carrying the 5 '-extension efficiently excluded EGFR-ex20_NNN primer from reaction (Fig. 5a,b), demonstrating robustness of the NOPE approach (see Fig. 5a,b). In the presence of TruSeq-short primer, inhibition of the target PCR amplification by NOPE-R1 and NOPE-R3 oligos was negligible, demonstrating that these oligos do not influence the efficiency of target PCR reaction (Fig. 5a, control, Fig. 5c, Fig. 6a). At the same time, NOPE-R2 notably inhibited target PCR, presumably interfering with the reaction in a sequence-specific manner. This indicates that newly designed NOPE oligo should be tested for side effects, as well as any new primer.

Simultaneously with the different NOPE oligos comparison we have determined their effective concentrations. The concentration of NOPE oligo should be sufficient to elongate nearly all molecules of the undesirable primer. At the same time, too high concentrations of the NOPE oligo could have an inhibiting effect on further amplification of target DNA. Increase of the NOPE oligos concentration from $0.1 \mu \mathrm{M}$ to $0.5 \mu \mathrm{M}$ consistently improved the efficiency of EGFR-ex20_NNN primer neutralisation (Fig. 5), while further increase of NOPE oligo concentration yielded poor returns.

To further verify universality of NOPE approach, we have additionally designed and tested NOPE-oligos for fragments of EGFR exons 18, 19, 21, and 22. All NOPE oligos demonstrated efficient exclusion of UMI-introducing primer at concentration of $0.5-1 \mu \mathrm{M}$ (Fig. 5d,e), with minimal inhibition of target PCR (Fig. 5f), thus confirming the general robustness of NOPE method.

\section{EGFR gene fragments library analysis by HTS}

Designed pipeline was applied to the EGFR-ex20 gene fragment library preparation procedure for the four circulating cell-free DNA samples of patients with lung cancer together with the control for assessment of NOPE-R3 efficiency. NOPE-R3 oligowas used at concentration $0.8 \mu \mathrm{M}$ for suppression of $0.1 \mu \mathrm{M}$ EGFR-ex20_NNN primer. Bands of anticipated size were observed for all samples. No PCR product was detected in control lane (without TruSeq-short primer in the 1st PCR). The final difference between the number of amplification cycles after three rounds of amplification for target samples and the control was 20-22. This internal control confirmed that addition of NOPE oligo removes the UMI-containing primer from the reaction very effectively, if not completely. 


\section{UMIs introduction via linear PCR}

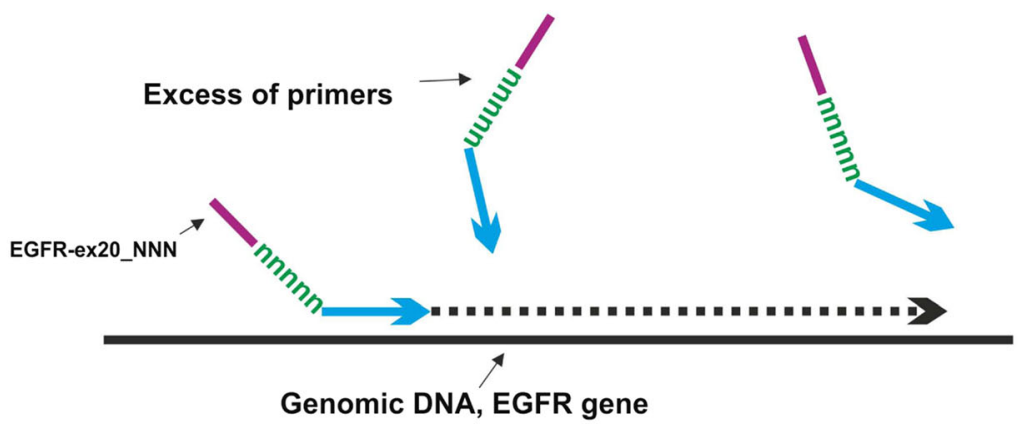

Neutralization of UMI-primer by NOPE oligo

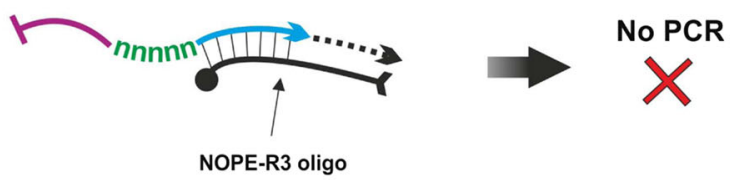

\section{Library amplification}

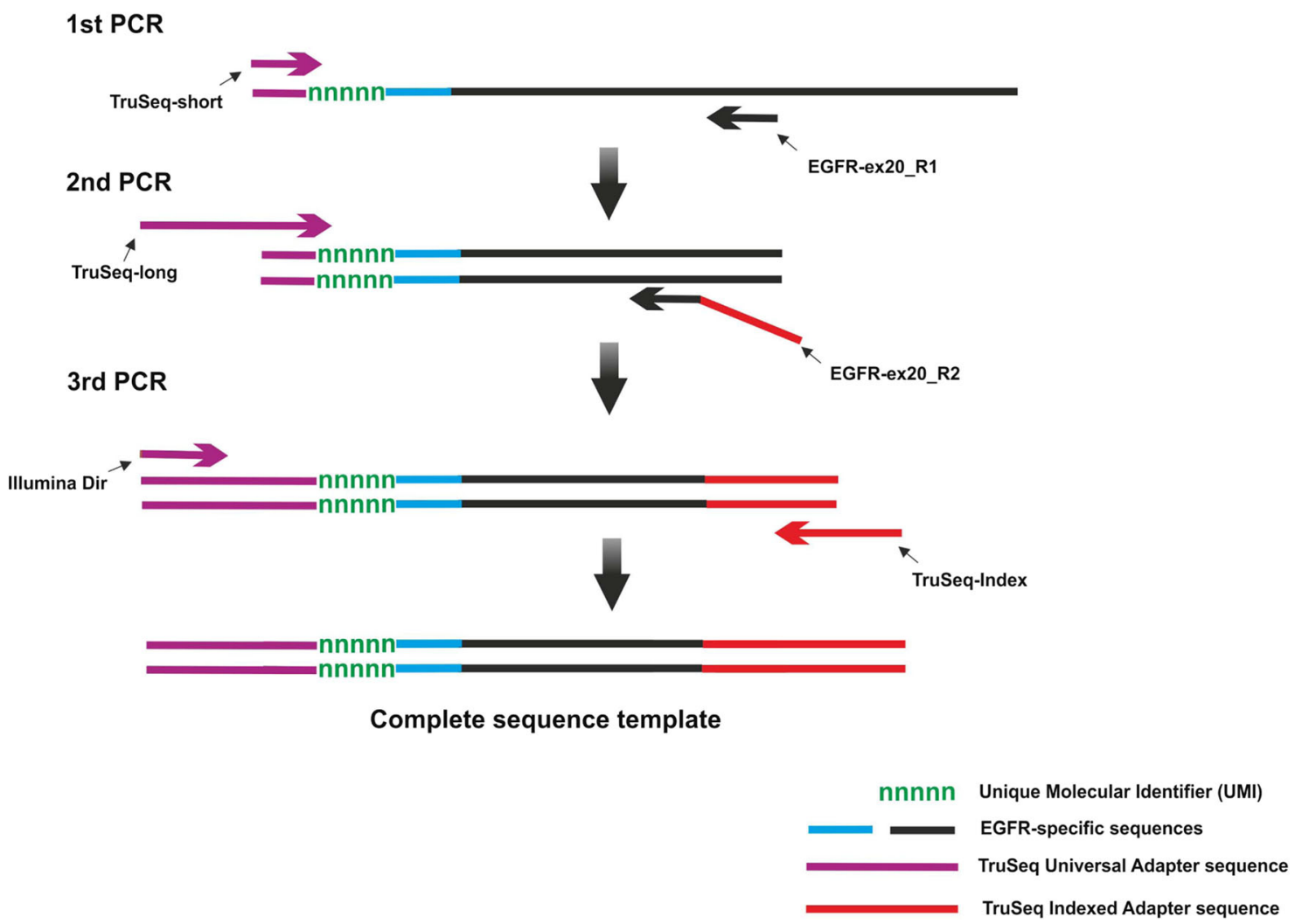

Fig. 2 (See legend on next page.) 
(See figure on previous page.)

Fig. 2 Exemplary experiment. DNA library construction and neutralization of UMI after PCR-based UMI introduction. Genomic DNA samples are amplified using 10 cycles of linear PCR with EGFR exon 20 specific primer modified to include UMI and partial Illumina sequencing adapter. Remaining UMI-primer is neutralized using 10 cycles of annealing/elongation in the presence of NOPE oligo. Next, 3 rounds of PCR are performed for amplification of EGFR gene fragments and introduction of complete Illumina adapters and indexes sequences

For comparison, we prepared the same EGFR-ex20 libraries using alternative method of EGFR-ex20_NNN primer elimination with E. coli Exonuclease I treatment followed by column purification (according to Ref. [3], with minor modifications, details on the method to be published elsewhere). $20 \mathrm{ng}$ of circulating cell-free DNA was used per each sample. 1/5 of the linear PCR product was used in case of NOPE. 1/ 2 of the column-purified linear PCR product was used in case of ExoI treatment. The resulting libraries of EGFR gene fragment were analyzed using pairedend Hiseq2500 Illumina sequencing.

Data analysis was performed using our MAGERI software designed to analyze UMI-tagged targeted genome re-sequencing data [23]. In this back-to-back comparison starting with identical amounts of DNA, NOPE method outperformed ExoI method in respect of the number of successfully labeled and amplified molecules (Fig. 7a), even in spite of the lower amount of the linear
PCR product used in the 1st PCR. This probably results from damage of template molecules by ExoI and material loss during purification step used after ExoI treatment.

At the same time, narrow shape of the UMI coverage peaks (Fig. 7b) confirms absence of notable inclusion of EGFR-ex20_NNN primer in PCR amplification after initial incorporation during linear PCR.

Next, we prepared EGFR-ex20 gene fragment libraries from the healthy PBMC DNA sample, standard reference genomic DNA (Tru-Q 7 1\% Tier reference mutation panel, Horizon Dx, USA; Cat. ID HD734), and the same reference DNA mixed with PBMC DNA in 1:9 proportion. Libraries were prepared by ExoI method and NOPE method, sequenced on Illumina HiSeq2500 and analyzed using MAGERI software. Both methods successfully identified mutation EGFR_T790 M present at frequency 0.01 in Tru-Q 7 1\% Tier reference mutation panel and at 0.001 frequency in reference panel mixed

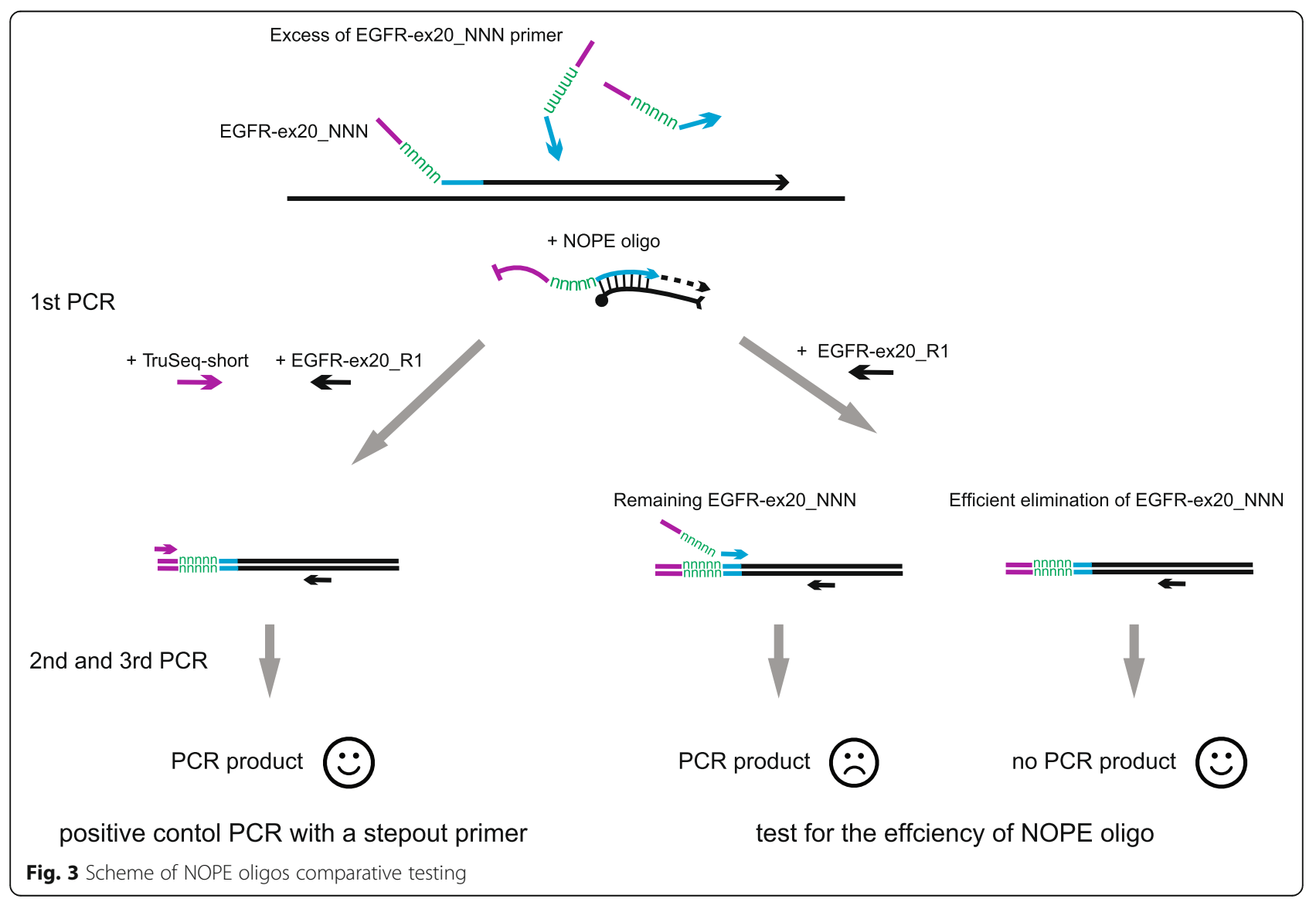


Table 1 Oligonucleotides

\begin{tabular}{|c|c|c|}
\hline Primer & Application & Sequence \\
\hline \multicolumn{3}{|c|}{$\begin{array}{l}\text { Primers for linear } \\
\text { PCR }\end{array}$} \\
\hline $\begin{array}{l}\text { EGFR- } \\
\text { ex20_NNN }\end{array}$ & \multirow{6}{*}{$\begin{array}{l}\text { PCR-based introduction of UMI } \\
\text { (UMI-containing primer), contains } \\
\text { partial sequence of TruSeq Illumina adapter }\end{array}$} & ACACGACGCTCTTCCGATCTNNNNNNNNNNNNNNCATCTGCCTCACCTCCACCGT \\
\hline $\begin{array}{l}\text { EGFR- } \\
\text { ex18- } \\
\text { 1reg_NNN }\end{array}$ & & ACACGACGCTCTTCCGATCTNNNNNNNNNNNNNNGAAGCTCCCAACCAAGCTC \\
\hline $\begin{array}{l}\text { EGFR- } \\
\text { ex19- } \\
\text { 1reg_NNN }\end{array}$ & & ACACGACGCTCTTCCGATCTNNNNNNNNNNNNNNCTGTCATAGGGACTCTGGAT \\
\hline $\begin{array}{l}\text { EGFR } \\
\text {-ex21_R_NNN }\end{array}$ & & ACACGACGCTCTTCCGATCTNNNNNNNNNNNNNNTCTTTCTCTTCCGCACCCAG \\
\hline $\begin{array}{l}\text { EGFR } \\
\text {-ex22_NNN }\end{array}$ & & ACACGACGCTCTTCCGATCTNNNNNNNNNNNNNNCATCCCAAGGTGCCTATCAA \\
\hline \multicolumn{2}{|l|}{ NOPE oligos } & \\
\hline NOPE-R1 & Neutralization of EGFR-ex20_NNN & GCACGCGTCGACGGTGGAGGTG-BHQ1 \\
\hline NOPE-R2 & & АCACGACGCTCTTCCGATCTACGGTGGAGGTGAGGCAG-BHQ1 \\
\hline NOPE-R3 & & GCACGCGTCGACGGTGGAGGTGAGGCAG-BHQ1 \\
\hline NOPE-RO & & ACGGTGGAGGTGAGGCAG-BHQ1 \\
\hline $\begin{array}{l}\text { NOPE-EGFR- } \\
\text { ex18 }\end{array}$ & Neutralization of EGFR-ex18-1reg_NNN & TCACACGTCTGAGCTTGGTTGGGAGCTTC-BHQ1 \\
\hline $\begin{array}{l}\text { NOPE-EGFR- } \\
\text { ex19 }\end{array}$ & $\begin{array}{l}\text { Neutralization of EGFR- } \\
\text { ex19-1reg_NNN }\end{array}$ & АTACTCTTCGATCCAGAGTCCCTATGACAG-BHQ1 \\
\hline $\begin{array}{l}\text { NOPE- EGFR- } \\
\text { ex21 }\end{array}$ & Neutralization of EGFR-ex21_R_NNN & CTTGACGTCACTGGGTGCGGAAGAGAAAGA-BHQ 1 \\
\hline $\begin{array}{l}\text { NOPE- EGFR- } \\
\text { ex22 }\end{array}$ & Neutralization of EGFR-ex22_NNN & АСТСTCATCCTTGATAGGCACCTTGGGATG-BHQ1 \\
\hline \multicolumn{3}{|l|}{$\begin{array}{l}\text { Primers for the } \\
1 \text { st PCR }\end{array}$} \\
\hline EGFR-ex20_R1 & Reverse nested EGFR-ex20-specific primer 1 & TGTTCCCGGACATAGTCCAG \\
\hline $\begin{array}{l}\text { EGFR-ex18- } \\
\text { 1reg_R1 }\end{array}$ & Reverse nested EGFR-ex18-specific primer 1 & AACGCACCGGAGCCCAGCACT \\
\hline $\begin{array}{l}\text { EGFR-ex19- } \\
\text { 1reg_R1 }\end{array}$ & Reverse nested EGFR-ex19-specific primer 1 & GATTTCCTTGTTGGCTTTCGGA \\
\hline EGFR-ex21_F1 & Forward nested EGFR-ex21-specific primer 1 & CTGGTGAAAACACCGCAGCA \\
\hline EGFR-ex22_R1 & Reverse nested EGFR-ex22-specific primer 1 & GCTCCAGACATCACTCTGGT \\
\hline TruSeq-short & Step-out primer 1 & TACACGACGCTCTTCCGATCT \\
\hline \multicolumn{3}{|l|}{$\begin{array}{l}\text { Primers for } \\
\text { 2nd PCR }\end{array}$} \\
\hline EGFR-ex20_R2 & $\begin{array}{l}\text { Reverse nested EGFR-ex20-specific primer 2, } \\
\text { contains partial sequence of TruSeq Illumina } \\
\text { adapter }\end{array}$ & GTGACTGGAGTTCAGACGTGTGCTCTTCCGATCTACATAGTCCAGGAGGCAGC \\
\hline $\begin{array}{l}\text { EGFR-ex18- } \\
\text { 1reg_R2 Br }\end{array}$ & $\begin{array}{l}\text { Reverse nested EGFR-ex18-specific primer 2, } \\
\text { contains partial sequence of TruSeq Illumina } \\
\text { adapter }\end{array}$ & GTGACTGGAGTTCAGACGTGTGCTCTTCCGATCTCGGAGCCCAGCACTTTGATC \\
\hline $\begin{array}{l}\text { EGFR-ex19- } \\
\text { 1reg_R2 Br }\end{array}$ & $\begin{array}{l}\text { Reverse nested EGFR-ex19-specific primer 2, } \\
\text { contains partial sequence of TruSeq Illumina } \\
\text { adapter }\end{array}$ & GTGACTGGAGTTCAGACGTGTGCTCTTCCGATCTTGTTGGCTTTCGGAGATGTT \\
\hline $\begin{array}{l}\text { EGFR-ex21_- } \\
\text { F2 Br }\end{array}$ & $\begin{array}{l}\text { Forward nested EGFR-ex21-specific primer 2, } \\
\text { contains partial sequence of TruSeq Illumina } \\
\text { adapter }\end{array}$ & GTGACTGGAGTTCAGACGTGTGCTCTTCCGATCTAAACACCGCAGCATGTCAAG \\
\hline $\begin{array}{l}\text { EGFR-ex22 } \\
\text { R2 Br }\end{array}$ & & GTGACTGGAGTTCAGACGTGTGCTCTTCCGATCTACATCACTCTGGTGGGTATAG \\
\hline
\end{tabular}


Table 1 Oligonucleotides (Continued)

\begin{tabular}{|c|c|c|}
\hline & $\begin{array}{l}\text { Reverse nested EGFR-ex22-specific primer 2, } \\
\text { contains partial sequence of TruSeq Illumina } \\
\text { adapter }\end{array}$ & \\
\hline TruSeq-long & $\begin{array}{l}\text { Step-out primer 2, contains sequence of } \\
\text { TruSeq Illumina adapter }\end{array}$ & AATGATACGGCGACCACCGAGATCTACACTCTTTCCCTACACGACGCTCTTCCGATCT \\
\hline \multicolumn{3}{|c|}{$\begin{array}{l}\text { Primers for } 3 r d \\
\text { PCR (introducing } \\
\text { Illumina indexes) }\end{array}$} \\
\hline TruSeq-Index1 & $\begin{array}{l}\text { Reverse step-out primer, anneals on the } \\
\text { EGFR-ex20_R2, contains complete sequence } \\
\text { of TruSeq Illumina adapter with sample index }\end{array}$ & CAAGCAGAAGACGGCATACGAGATCGTGATGTGACTGGAGTTCAGACGTGT \\
\hline Illumina-Dir & Forward step-out primer 3 & AATGATACGGCGACCACCGAGATC \\
\hline \multicolumn{3}{|c|}{ Real time PCR probes } \\
\hline Z-IGFR ex20 & Real time PCR probe for exon 20. & Fam-AGCTCATCACGCAGCTCATGCCCTT-BHQ1 \\
\hline Z-TruUni & $\begin{array}{l}\text { Universal real time PCR probe for TuSeq } \\
\text { adapter }\end{array}$ & Fam-АСАСТСТTTCССТАCACGACGCTCTT-BHQ1 \\
\hline
\end{tabular}

1:9 with a healthy donor PBMC (Fig. 7c). These results demonstrate applicability and efficiency of NOPE method for targeted re-sequencing with UMI.

\section{Discussion}

Today UMI approach is widely used in multiple applications, including genome, transcriptome [2, 3, 24] and microbiome [9] analysis, immune repertoires sequencing [25-32], detection of low-frequency mutations [3, 33], studying the incidence of transcription [34], primer synthesis [3], or sequencing [35] errors.

PCR-based introduction of UMI may use target-specific primers modified to include UMI and Illumina sequencing adaptors. The resulting PCR product is a sequencingready library that does not require additional adaptors ligation step thus reducing the overall cost, time and complexity of the experiment. Here we focused on further rationalization of UMI-labeled library preparation for targeted resequencing and described application of NOPE method for processing of DNA library with PCR-based UMI introduction.

We showed that addition of NOPE oligo efficiently resolves the problem of UMI-containing primers remaining after PCR-based introduction of molecular identifiers. We implemented this technology in our current HTS project related to the tumor mutations profiling. On the exemplary system we described library preparation procedure for targeted resequencing and cancer mutation detection in single EGFR gene. However, the same method could be easily adapted for multiple genes library preparation.

Advantages of the proposed method become critical when it comes to the low-copy number DNA samples. Thus from a practical perspective, NOPE oligos can be widely applied in all those cases where purification of reaction may result in material loss. In particular, NOPE method should be efficient for molecular assessment of circulating cell-free tumor or fetal DNA, or minor cell samples of interest.

EGFR gene exon 20

GTGATGGCCAGCGTGGACAACCCCCACGTGTGCCGCCTGCTGGGCATCTGCCTCACCTCCACCGTGCAGCTCATCACGCAGCTCATGCCCT

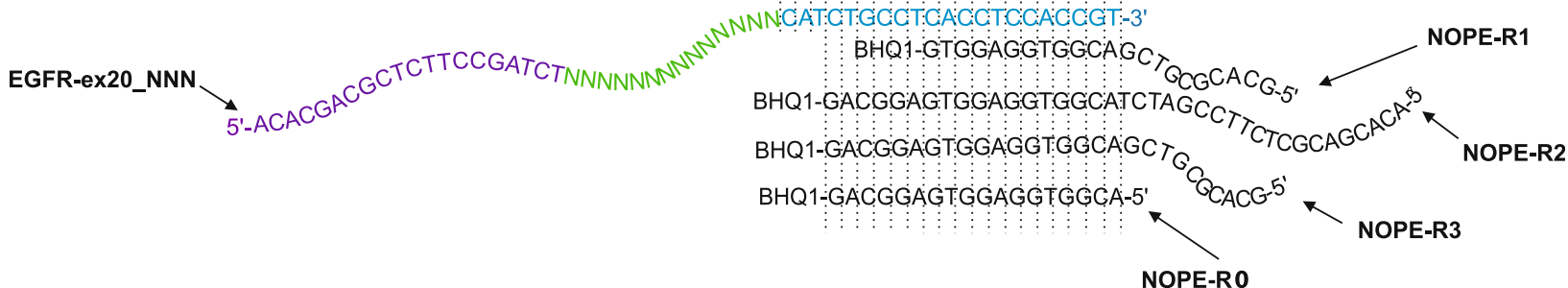

Fig. 4 EGFR gene region of interest. Region of human EGFR gene sequence showing the location of the UMI-containing primer EGFR-ex20_NNN and 4 different NOPE oligos 
a
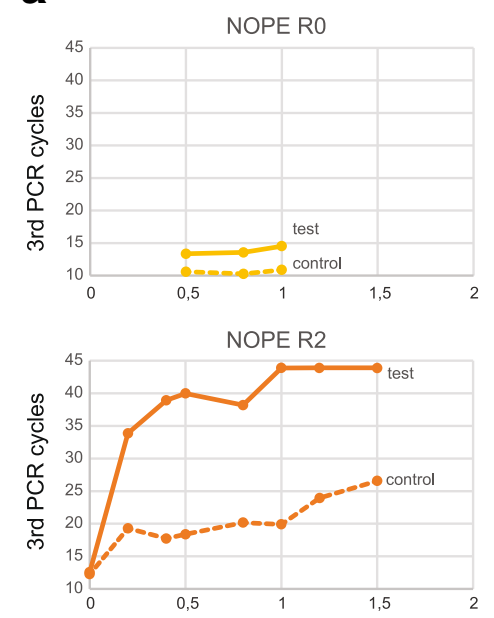

NOPE oligo concentration, $\mu \mathrm{M}$

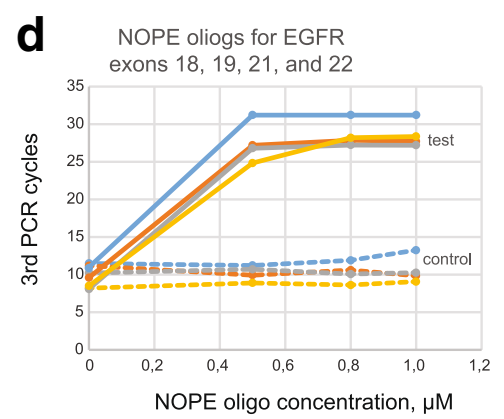

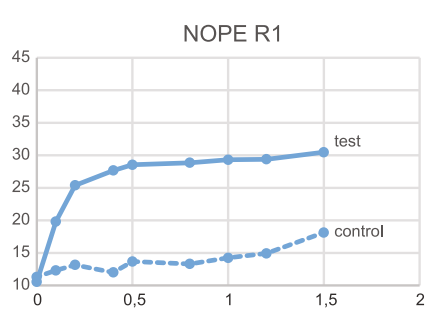

NOPE R3

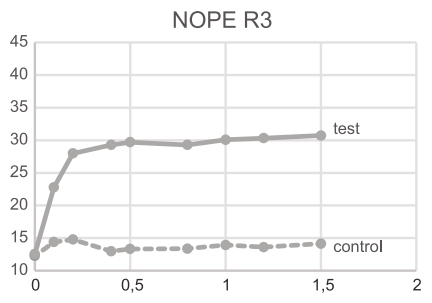

NOPE oligo concentration, $\mu \mathrm{M}$

e

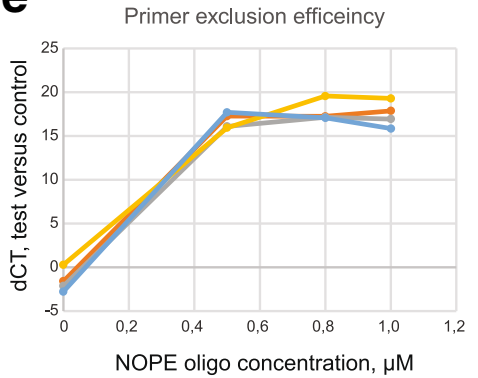

b

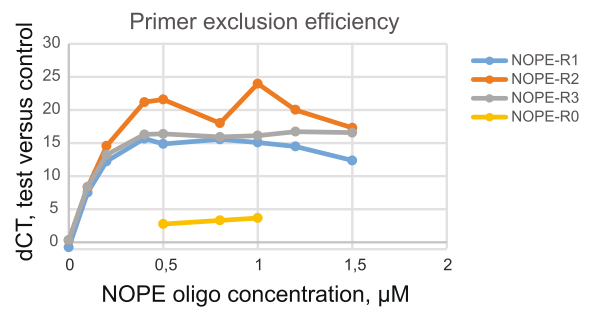

C

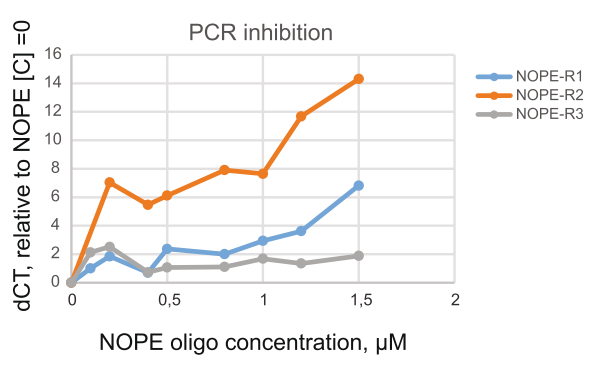

f

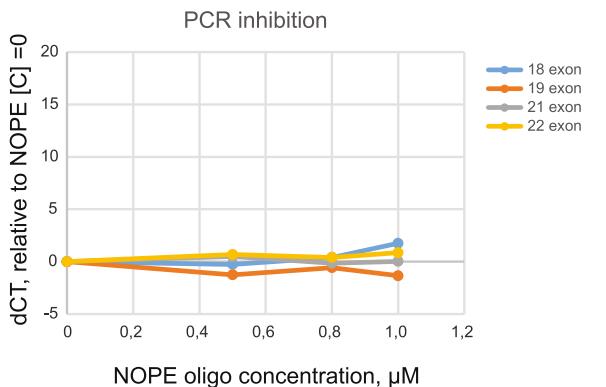

Fig. 5 Real-time PCR analysis of NOPE oligos efficiency. Results of the 3rd PCR are shown. Typical results are shown for the two independent experiments each performed in two replicas. See Table 1 for all oligonucleotides used. a Four different NOPE oligos (NOPE-R0, R1, R2, R3) performance in amplification of EGFR gene exon 20 fragment. Dotted lines represent positive control reaction containing NOPE oligo and two amplification primers: forward TruSeq-short and reverse EGFR-ex20-R1 in the 1st PCR. Solid lines represent test reactions containing NOPE oligo and only reverse primer EGFR-ex20_R1 in the 1st PCR. b Comparison of EGFR-NNN-ex20 primer exclusion efficiency by the four NOPE oligos. dCt between control reaction with two amplification primers in the 1st PCR and test reaction with only reverse primer in the 1st PCR reflects efficiency of EGFR-NNN-ex20 primer exclusion. c Comparison of the NOPE oligos inhibitory effect on amplification of target EGFR gene exon 20 fragment. dCt between amplification with two EGFR exon 20 amplification primers with and without NOPE oligo reflects inhibition of the target DNA amplification by NOPE oligo. d NOPE oligos performance in amplification of EGFR gene exons 18, 19, 21, and 22 fragments. Dotted lines represent control reactions containing NOPE oligo and two amplification primers in the 1st PCR: TruSeq-short and complementary EGFR primer. Solid lines represent test reactions containing NOPE oligo and a single EGFR primer in the 1st PCR. e EGFR-NNN primers exclusion efficiency by NOPE oligos for EGFR gene exons 18, 19, 21, and 22 fragments. dCt between control reaction with two amplification primers in the 1st PCR and test reaction with only the reverse primer in 1st PCR reflects efficiency of of EGFR-NNN primer exclusion. $\mathbf{f}$ NOPE oligos inhibitory effect on amplification of target fragments of EGFR gene exons 18, 19, 21, and 22. dCt between reactions containing NOPE oligo and two EGFR amplification primers and reactions containing only EGFR amplification primers reflects inhibition of the target DNA amplification by NOPE oligo

Furthermore, NOPE method offers avenues in various fields beyond HTS. For example, this approach could be used for nested PCR free of interim dilution and purification steps. Inhibition of the 1st PCR primers by NOPE oligos added right to the 1st PCR mixture allows to proceed with the 2nd PCR in the same tube just by adding of nested primers. Another applicational example is inhibition of megaprimers in those particular cases where mega-priming process may adversely affect subsequent reactions [20, 21]. Other applications may be invented. We believe that simplicity and robustness of the NOPE method should make it popular in various PCR protocols.

\section{Conclusions}

In the present study, we implemented our NOPE method for the UMI-based library preparation for HTS. Our results show that NOPE approach allows to achieve nearly complete elimination of remaining UMI-containing primers from the reaction without cleanup step and thus testify the utility of NOPE 
a

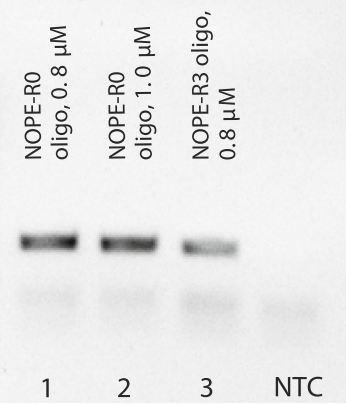

b

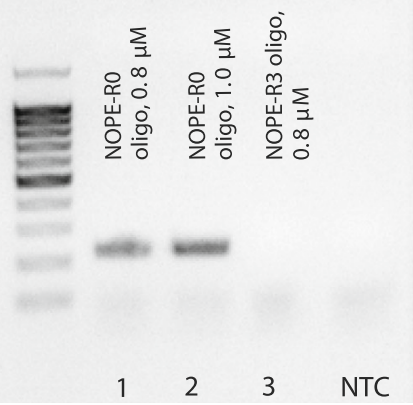

Fig. 6 Comparison of NOPE-R3 and NOPE-RO oligo performance. Lanes A1-A3 represent reactions containing two primers in the 1st PCR forward TruSeq-short and reverse EGFR-ex20-R1; lanes B1-B3 represent reactions containing only the reverse primer EGFR-ex20-R1. NTC - non template control. See Methods for details. Note absence of PCR product in B3 reaction that has been amplified for 11 more cycles compared to A3 and 9 more cycles compared to B1

oligos in HTS analyses, especially where low-copy number DNA samples may compromise the results.

\section{Methods}

\section{UMI-barcoded library preparation using NOPE oligo}

Specimens collection and DNA extraction. Samples of healthy donors PMBC DNA, circulating cell-free DNA, and reference genomic DNA (Tru-Q 7 (1.3\% Tier) Reference Standard, Horizon) were used in experiments. Circulating cell-free DNA was isolated from the blood plasma of patients with adenocarcinoma at Molecular Biology \& Cytogenetics Lab, Russian Center for Roentgenology \& Radiology (Moscow, Russian Federation). DNA extraction was performed with the use of QIAamp Circulating Nucleic Acid Kit (Qiagen).
Introduction of UMI via linear PCR. $25 \mathrm{ng}$ of gDNA was used for the reaction. Besides DNA, linear PCR $(50 \mu \mathrm{L})$ contained also: $1 \times$ Tersus Buffer (Evrogen), $200 \mu \mathrm{M}$ of each dNTP, $5 \mathrm{U}$ of Tersus polymerase (Evrogen) and $0.1 \mu \mathrm{M}$ of the forward primer EGFRex20_NNN. Thermal cycling conditions were: $1 \mathrm{~min}$ at $95{ }^{\circ} \mathrm{C}$; 10 cycles of $15 \mathrm{~s}$ at $95{ }^{\circ} \mathrm{C}, 20 \mathrm{~s}$ at $60{ }^{\circ} \mathrm{C}, 30 \mathrm{~s}$ at $72{ }^{\circ} \mathrm{C}$; and finally $2 \mathrm{~min}$ at $72{ }^{\circ} \mathrm{C}$. Nontemplate control was included.

Neutralization of remaining EGFR-ex20_NNN and NOPE oligo concentration optimization. For neutralization of remaining UMI-containing primers after introduction of UMI into EGFR gene fragments, $0.1-1.5 \mu \mathrm{M}$ of four different NOPE oligos were added to the reactions. NOPE oligos concentrations and NOPE oligo/
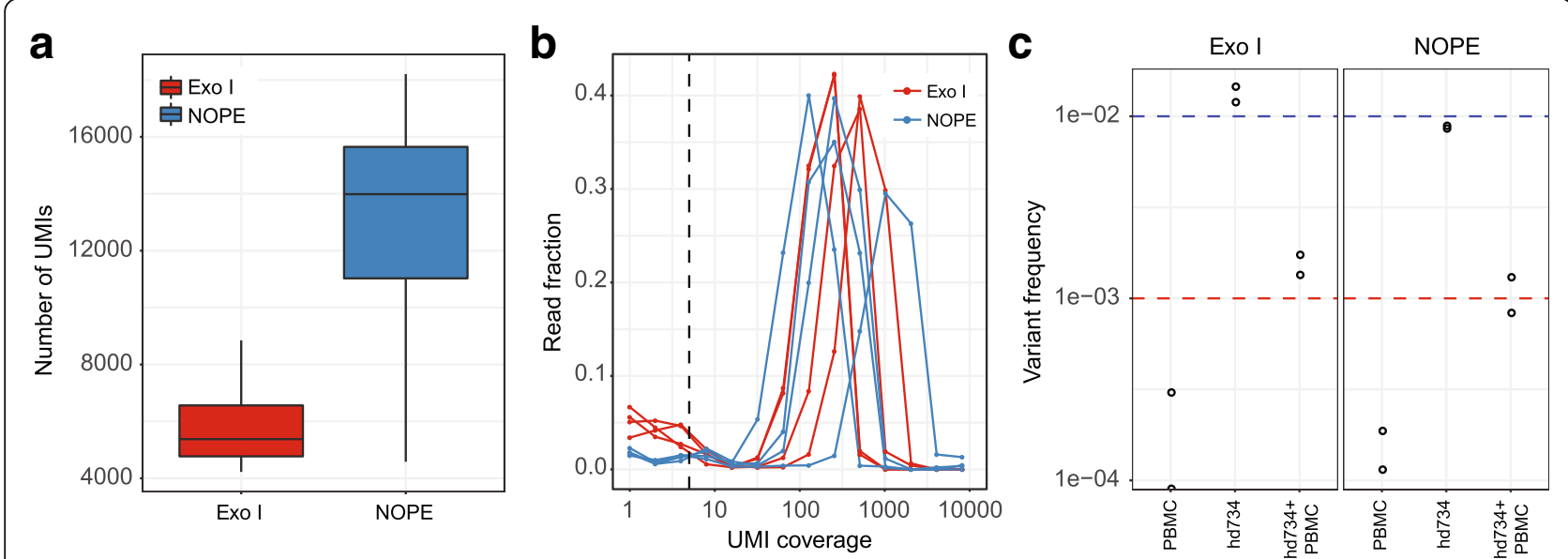

Fig. 7 HTS data analysis. EGFR-ex20 gene libraries were prepared in parallel using NOPE and Exol approaches for EGFR-ex20_NNN primer exclusion. $\mathbf{a}$ The number of detected UMI sequences with a sufficient (5+ read) coverage. $\mathbf{b}$ UMI coverage distribution showing the fraction of reads ( $Y$ axis) tagged with UMls covered $X$ times. Dashed line shows reads-per-UMl threshold selected for data analysis. c Identification of T790 M mutation in EGFR gene in control PBMC DNA, the reference standard and their 9:1 mixture. Red and blue lines show the expected mutation frequency in the mixture and pure reference standard sample, respectively 
Table 2 NOPE oligo quantities tested during concentration optimization

\begin{tabular}{|c|c|c|c|c|c|c|c|c|}
\hline № of sample & 1 & 2 & 3 & 4 & 5 & 6 & 7 & 8 \\
\hline NOPE-R3/EGFR-ex20_NNN ratio & $1: 1$ & $2: 1$ & $4: 1$ & $5: 1$ & $8: 1$ & $10: 1$ & 12:1 & $15: 1$ \\
\hline NOPE-R3 oligo concentration, $\mu \mathrm{M}$ & 0.1 & 0.2 & 0.4 & 0.5 & 0.8 & 1.0 & 1.2 & 1.5 \\
\hline
\end{tabular}

EGFR-ex20_NNN primer ratios used in the experiment are shown in Table 2. The temperature settings were: 10 cycles of $30 \mathrm{~s}$ at $60{ }^{\circ} \mathrm{C}, 20 \mathrm{~s}$ at $65^{\circ} \mathrm{C}$.

\section{Library amplification}

1st PCR. After neutralization of UMI-containing primer, reactions were divided into two halves. For further libraries amplification, $0.2 \mu \mathrm{M}$ of the forward primer TruSeqshort and $0.2 \mu \mathrm{M}$ of the reverse primer EGFR-ex20_R1 were added to the positive control half of the reaction. Only reverse primer EGFR-ex20_R1 was added to the test half of the reaction (Fig. 3). The following cycle settings were used: 30 cycles of $15 \mathrm{~s}$ at $95{ }^{\circ} \mathrm{C}, 20 \mathrm{~s}$ at $62{ }^{\circ} \mathrm{C}$ and $30 \mathrm{~s}$ at $72{ }^{\circ} \mathrm{C}$, final extension for $2 \mathrm{~min}$ at $72{ }^{\circ} \mathrm{C}$.

2nd PCR. The second amplification was carried out using $1 \mu \mathrm{L}$ of unpurified 1000-fold diluted product of the 1st PCR. $25 \mu \mathrm{L}$ reaction contained: $1 \times$ Tersus Buffer, $200 \mu \mathrm{M}$ of each dNTP, $5 \mathrm{U}$ of Tersus polymerase, $0.2 \mu \mathrm{M}$ of the forward primer TruSeq-long and $0.2 \mu \mathrm{M}$ of the reverse primer EGFR-ex20_R2. The following cycle settings were used: denaturation, $1 \mathrm{~min}$ at $95{ }^{\circ} \mathrm{C}$, followed by 11 cycles of $15 \mathrm{~s}$ at $95{ }^{\circ} \mathrm{C}, 20 \mathrm{~s}$ at $63^{\circ} \mathrm{C}$ and $30 \mathrm{~s}$ at $72{ }^{\circ} \mathrm{C}$. Final extension was $2 \mathrm{~min}$ at $72{ }^{\circ} \mathrm{C}$.

3rd PCR. During the third amplification Illumina indexes were incorporated into DNA library. 3rd amplification was carried out using $2 \mu \mathrm{L}$ of unpurified 1000fold diluted product of the 2nd PCR. $25 \mu \mathrm{L}$ reaction contained: $1 \times$ qPCRmix-HS (Evrogen), $0.2 \mu \mathrm{M}$ of the Illumina-Dir primer, $0.2 \mu \mathrm{M}$ of the TruSeq-Index 1 primer and $0.2 \mu \mathrm{M}$ of the TaqMan probe. Real-time PCR was performed using ABI PRISM 7500 Sequence Detection System (Applied Biosystems). Reactions were set up in duplicate in two independent experiments. The following cycle settings were used: 3 min of polymerase activation at $95{ }^{\circ} \mathrm{C}$, followed by 45 cycles of $15 \mathrm{~s}$ at $95^{\circ} \mathrm{C}$, $30 \mathrm{~s}$ at $63{ }^{\circ} \mathrm{C}$ and $1 \mathrm{~min}$ at $72{ }^{\circ} \mathrm{C}$. Fluorescence signal was collected at every annealing step.

Testing of NOPE oligos for EGFR exons 18, 19, 21, 22 was performed under the same reaction conditions that have been described above for EGFR exon 20.

\section{Comparison of NOPE-R3 and NOPE-RO oligo performance (Fig. 6)}

Non-template control was included. After UMI-containing primer neutralization step, all reactions were divided into two halves. To the one half of reaction, $0.1 \mu \mathrm{M}$ of the forward primer TruSeq-short and $0.1 \mu \mathrm{M}$ of the reverse primer EGFR-ex20_R1 were added in the 1st PCR mix. To the second half, only $0.1 \mu \mathrm{M}$ of the reverse primer EGFRex20_R1 was added in 1st PCR mix. 30 amplification cycles were performed for all samples during 1st PCR. The following cycles number were used during 2nd PCR: for A1, A2, A3, B1, B2-11; for B3-20. The following number of PCR cycles was used during the 3rd PCR: for A1, A2, A3-11; for B1, B2, B3-13. The resulting PCR product was visualized on agarose gel.

\section{Abbreviations}

BHQ1: Black hole quencher 1; EGFR: Epidermal growth factor receptor; HTS: High-throughput sequencing; LNA: Locked nucleic acids; NOPE: Nonsense-mediated primer exclusion - method reported here; NSCLC: Non-small cell lung cancer; PBMC: Peripheral blood mononuclear cells; PCR: Polymerase chain reaction; PNA: Peptide nucleic acids; SPRI: Solid phase reversible immobilization; UMI: Unique molecular identifiers

\section{Acknowledgements}

Not applicable.

Availability of data and materials

Raw sequencing data for the experiment shown on Fig. 7 are deposited in NCBI SRA database (PRJNA369461).

\section{Funding}

This work was supported by Russian Science Foundation project №14-1400533, Ministry of Education, Youth and Sports of the Czech Republic under the project CEITEC 2020 (LQ1601), and European Union's Horizon 2020 research and innovation program under grant agreement No 633592 (APERIM, this publication reflects only the author's view and the Commission is not responsible for any use that may be made of the information it contains). The funders had no role in study design, data collection and analysis, decision to publish, or preparation of the manuscript. Experiments were carried out in part using equipment provided by the ShemyakinOvchinnikov Institute of Bioorganic Chemistry Core Facility.

\section{Authors' contributions}

MAT, DAB and DMC invented the NOPE method. MAT, DAS, IAS, ARZ, and OIZ performed the experiments. MS performed data analysis. SL and DMC supervised the work. MAT, DAB and DMC worked on the manuscript. All authors read and approved the final manuscript.

\section{Competing interests}

The authors declare that they have no competing interests.

\section{Consent for publication}

Not applicable.

\section{Ethics approval and consent to participate}

Circulating cell-free DNA was isolated from the blood plasma of patients with adenocarcinoma at Molecular Biology \& Cytogenetics Lab, Russian Center for Roentgenology \& Radiology (Moscow, Russian Federation). The study was approved by the ethics committee of Russian Center for Roentgenology \& Radiology and conducted in accordance with the Declaration of Helsinki. All donors were informed of the final use of the samples and signed an informed consent document.

\section{Publisher's Note}

Springer Nature remains neutral with regard to jurisdictional claims in published maps and institutional affiliations. 


\section{Author details}

'Pirogov Russian National Research Medical University, Moscow, Russia. ${ }^{2}$ Shemiakin-Ovchinnikov Institute of Bioorganic Chemistry, Russian Academy of Science, Moscow, Russia. ${ }^{3}$ Evrogen JSC, Moscow, Russia. ${ }^{4}$ Central European Institute of Technology, Masaryk University, Brno, Czech Republic. ${ }^{5}$ Skolkovo Institute of Science and Technology, Moscow, Russia.

Received: 1 August 2016 Accepted: 24 May 2017

Published online: 05 June 2017

\section{References}

1. Casbon JA, Osborne RJ, Brenner S, Lichtenstein CP. A method for counting PCR template molecules with application to next-generation sequencing. Nucleic Acids res. 2011;39(12):e81.

2. Kivioja T, Vaharautio A, Karlsson K, Bonke M, Enge M, Linnarsson S, et al. Counting absolute numbers of molecules using unique molecular identifiers. Nat Methods. 2012:9(1):72-4.

3. Kinde I, Wu J, Papadopoulos N, Kinzler KW, Vogelstein B. Detection and quantification of rare mutations with massively parallel sequencing. Proc Natl Acad Sci U S a. 2011;108(23):9530-5.

4. Diehl F, Schmidt K, Durkee KH, Moore KJ, Goodman SN, Shuber AP, et al, Analysis of mutations in DNA isolated from plasma and stool of colorectal cancer patients. Gastroenterology. 2008;135(2):489-98.

5. Fan HC, Blumenfeld YJ, Chitkara U, Hudgins L, Quake SR. Noninvasive diagnosis of fetal aneuploidy by shotgun sequencing DNA from maternal blood. Proc Natl Acad Sci U S a. 2008;105(42):16266-71.

6. Burrell RA, Swanton $C$. The evolution of the unstable cancer genome. Curr Opin Genet dev. 2014;24:61-7.

7. Colman RE, Schupp JM, Hicks ND, Smith DE, Buchhagen JL, Valafar F, et al. Detection of low-level mixed-population drug resistance in mycobacterium tuberculosis using high Fidelity amplicon sequencing. PLoS One. 2015;10(5): e0126626.

8. Van Laethem K, Theys K, Vandamme AM. HIV-1 genotypic drug resistance testing: digging deep, reaching wide? Current Opinion in Virology. 2015;14:16-23.

9. Faith JJ, Guruge JL, Charbonneau M, Subramanian S, Seedorf H, Goodman AL, et al. The long-term stability of the human gut microbiota. Science. 2013; 341(6141):1237439

10. Barrick JE, Lenski RE. Genome dynamics during experimental evolution. Nat rev Genet. 2013;14(12):827-39.

11. Carlson CM, Dupuy AJ, Fritz S, Roberg-Perez KJ, Fletcher CF, Largaespada DA. Transposon mutagenesis of the mouse germline. Genetics. 2003;165(1):243-56.

12. Corless CL, Harrell P, Lacouture M, Bainbridge T, Le C, Gatter K, et al. Allelespecific polymerase chain reaction for the imatinib-resistant KIT D816V and D816F mutations in mastocytosis and acute myelogenous leukemia. The Journal of Molecular Diagnostics : JMD. 2006;8(5):604-12.

13. Peano C, Lesignoli F, Gulli M, Corradini R, Samson MC, Marchelli R, et al. Development of a peptide nucleic acid polymerase chain reaction clamping assay for semiquantitative evaluation of genetically modified organism content in food. Anal Biochem. 2005;344(2):174-82.

14. Karkare S, Bhatnagar D. Promising nucleic acid analogs and mimics: characteristic features and applications of PNA, LNA, and morpholino. Appl Microbiol Biotechnol. 2006;71(5):575-86.

15. Tatsumi K, Mitani Y, Watanabe J, Takakura H, Hoshi K, Kawai Y, et al. Rapid screening assay for KRAS mutations by the modified smart amplification process. The Journal of Molecular Diagnostics : JMD. 2008;10(6):520-6.

16. Orum H. PCR clamping. Current Issues in Molecular Biology. 2000;2(1):27-30.

17. Troedsson C, Lee RF, Walters T, Stokes V, Brinkley K, Naegele V, et al. Detection and discovery of crustacean parasites in blue crabs (Callinectes sapidus) by using 18S rRNA gene-targeted denaturing high-performance liquid chromatography. Appl Environ Microbiol. 2008;74(14):4346-53.

18. Dominguez PL, Kolodney MS. Wild-type blocking polymerase chain reaction for detection of single nucleotide minority mutations from clinical specimens. Oncogene. 2005;24(45):6830-4.

19. Boessenkool S, Epp LS, Haile J, Bellemain E, Edwards M, Coissac E, et al. Blocking human contaminant DNA during PCR allows amplification of rare mammal species from sedimentary ancient DNA. Mol Ecol. 2012;21 (8):1806-15.

20. Turchaninova MA, Britanova OV, Bolotin DA, Shugay M, Putintseva EV, Staroverov DB, et al. Pairing of T-cell receptor chains via emulsion PCR. Eur J Immunol. 2013;43(9):2507-15.
21. Munson DJ, Egelston CA, Chiotti KE, Parra ZE, Bruno TC, Moore BL, et al. Identification of shared TCR sequences from T cells in human breast cancer using emulsion RT-PCR. Proc Natl Acad Sci U S a. 2016;113(29):8272-7.

22. Pao W, Miller VA, Politi KA, Riely GJ, Somwar R, Zakowski MF, et al. Acquired resistance of lung adenocarcinomas to gefitinib or erlotinib is associated with a second mutation in the EGFR kinase domain. PLoS med. 2005;2(3):e73.

23. Shugay M, Zaretsky AR, Shagin DA, Shagina IA, Volchenkov IA, Shelenkov AA, et al. MAGERI: computational pipeline for molecular-barcoded targeted resequencing. PLoS Comput Biol. 2017;13(5):e1005480.

24. Grun D, Kester L, van Oudenaarden A. Validation of noise models for singlecell transcriptomics. Nat Methods. 2014;11(6):637-40.

25. Vollmers C, Sit RV, Weinstein JA, Dekker CL, Quake SR. Genetic measurement of memory B-cell recall using antibody repertoire sequencing. Proc Natl Acad Sci U S a. 2013;110(33):13463-8.

26. Britanova OV, Putintseva EV, Shugay M, Merzlyak EM, Turchaninova MA, Staroverov DB, et al. Age-related decrease in TCR repertoire diversity measured with deep and normalized sequence profiling. J Immunol. 2014; 192(6):2689-98.

27. Shugay M, Britanova OV, Merzlyak EM, Turchaninova MA, Mamedov IZ, Tuganbaev TR, et al. Towards error-free profiling of immune repertoires. Nat Methods. 2014;11(6):653-5.

28. He L, Sok D, Azadnia P, Hsueh J, Landais E, Simek M, et al. Toward a more accurate view of human B-cell repertoire by next-generation sequencing, unbiased repertoire capture and single-molecule barcoding. Scientific Reports. 2014;4:6778.

29. Egorov ES, Merzlyak EM, Shelenkov AA, Britanova OV, Sharonov GV, Staroverov DB, et al. Quantitative profiling of immune repertoires for minor lymphocyte counts using unique molecular identifiers. J Immunol. 2015; 194(12):6155-63.

30. Khan TA, Friedensohn S, de Vries AR, Straszewski J, Ruscheweyh HJ, Reddy ST. Accurate and predictive antibody repertoire profiling by molecular amplification fingerprinting. Sci adv. 2016;2(3):e1501371.

31. Feng Y, van der Veeken J, Shugay M, Putintseva EV, Osmanbeyoglu HU, Dikiy S, et al. A mechanism for expansion of regulatory T-cell repertoire and its role in self-tolerance. Nature. 2015;528(7580):132-6.

32. Britanova OV, Shugay M, Merzlyak EM, Staroverov DB, Putintseva EV, Turchaninova MA, et al. Dynamics of individual T cell repertoires: from cord blood to centenarians. J Immunol. 2016;196(12):5005-13.

33. Tie J, Kinde I, Wang Y, Wong HL, Roebert J, Christie M, et al. Circulating tumor DNA as an early marker of therapeutic response in patients with metastatic colorectal cancer. Ann Oncol. 2015;26(8):1715-22.

34. Gout JF, Thomas WK, Smith Z, Okamoto K, Lynch M. Large-scale detection of in vivo transcription errors. Proc Natl Acad Sci U S a. 2013;110(46):18584-9.

35. Deakin $C T$, Deakin JJ, Ginn SL, Young P, Humphreys D, Suter CM, et al. Impact of next-generation sequencing error on analysis of barcoded plasmid libraries of known complexity and sequence. Nucleic Acids res. 2014;42(16):e129.

\section{Submit your next manuscript to BioMed Central and we will help you at every step:}

- We accept pre-submission inquiries

- Our selector tool helps you to find the most relevant journal

- We provide round the clock customer support

- Convenient online submission

- Thorough peer review

- Inclusion in PubMed and all major indexing services

- Maximum visibility for your research

Submit your manuscript at www.biomedcentral.com/submit
Biomed Central 